\title{
Atomic Resolution Tomography Reconstruction of Tilt Series based on a GPU Accelerated Hybrid Input-Output Algorithm using Polar Fourier Transform
}

\author{
Xiangwen Lu, 1,2,a Wenpei Gao,2,3,a Jian-Min Zuo,2,3,* Jiabin Yuan, ${ }^{1}$ \\ ${ }^{1}$ College of Computer Science and Technology, Nanjing University of Aeronautics and Astronautics, Nanjing 210016, China \\ ${ }^{2}$ Department of Materials Science and Engineering, University of Illinois at Urbana-Champaign, 1304 W. Green St., \\ Urbana, IL 61801, USA \\ ${ }^{3}$ Materials Research Laboratory, University of Illinois at Urbana-Champaign, 1304 W. Green St., Urbana, IL 61801, USA \\ ${ }^{*}$ Corresponding author. Tel: +1 217-244-6504; Fax: +1 217-333-2736. E-mail address: jianzuo@illinois.edu \\ a These two authors contributed equally to this work
}

\begin{abstract}
Advances in diffraction and transmission electron microscopy (TEM) have greatly improved the prospect of three-dimensional (3D) structure reconstruction from two-dimensional (2D) images or diffraction patterns recorded in a tilt series at atomic resolution. Here, we report a new GPU (graphics processing unit) accelerated iterative transformation algorithm (ITA) based on polar fast Fourier transform for reconstructing 3D structure from $2 \mathrm{D}$ diffraction patterns. The algorithm also applies to image tilt series by calculating diffraction patterns from the recorded images using the projection-slice theorem. A gold icosahedral nanoparticle of 309 atoms is used as the model to test the feasibility, performance and robustness of the developed algorithm using simulations. Atomic resolution in 3D is achieved for the 309 atoms Au nanoparticle using 75 diffraction patterns covering $150^{\circ}$ rotation. The capability demonstrated here provides an opportunity to uncover the $3 \mathrm{D}$ structure of small objects of nanometers in size by electron diffraction.
\end{abstract}

Key words: Three dimensional imaging, Diffraction imaging, TEM tomography, Phase retrieval algorithms, Atomic resolution, GPU accelerated, Polar Fourier transform

\section{Introduction}

Three-dimensional reconstruction at atomic resolution is a major challenge in materials structure characterization. Recent advances in electron optics have enabled a direct determination of atomic structure at sub-Ångstrom resolution in 2D projection [1-4]. However, imaging atoms inside, and at the surface of, a nanoparticle or determining the structure of a 3D defect requires information of the 3D atomic structure. Tomographic reconstruction at atomic resolution provides a possible way forward to 3D structure determination for any objects.

At atomic resolution, several groups have reported 3D reconstruction using Z-contrast images obtained in a scanning transmission electron microscope (STEM) equipped with an annular dark field (ADF) detector. Van Aert et al. reported a successful reconstruction of the $3 \mathrm{D}$ atomic structure of $\mathrm{Ag}$ precipitates in the $\mathrm{Al}$ matrix using discrete tomography [5]. This method only requires electron images recorded in a few zone-axis projections, but a prior knowledge of the structure is necessary for the reconstruction. The other approach is to detect atomic position in 3D using the STEM depth sectioning method [6-8] or using a combination of quantitative STEM and multislice simulation [9]. The resolution of these methods, however, is limited along the beam direction by the probe elongation effect resulting from the small electron beam convergence angle and by electron multiple scattering along atomic columns [6, 10]. Recently, Jianwei Miao's group at UCLA demonstrated the 3D reconstruction of an Au nanoparticle at $2.4 \AA$ resolution by using tomographic reconstruction based on the so-called equal-sloped fast Fourier Transform [11]. The reconstruction is based on the Z-contrast image data recorded in a tilt series from $-72.6^{\circ}$ to $72.6^{\circ}$ in equal-slope increment. This method has been further applied to image dislocations in an Au nanoparticle in 3D [12].

Here we report a new method for 3D tomographic reconstruction based on the hybrid input-output (HIO) algorithm developed by Fienup [13] and polar Fourier fast Transform (FFT). Only diffraction data obtained in a tilt series are used for reconstruction. Because information recorded in a diffraction pattern is limited only by scattering, the algorithm reported here has the potential to achieve the highest resolution. Another major application of this method is in tomography where the projected object functions, instead of diffraction patterns, are recorded directly in real space. Using the projection-slice theorem, Fourier transform (FT) of the projected object function gives the diffraction pattern with information up to the image resolution. By using only amplitudes of FT, our method does not require alignment of projected object functions, which has to be done at the precision of $\AA$ for atomic resolution tomography and thus provides a major advantage over regular tomographic methods.

We use the HIO algorithm originally proposed by Fienup to retrieve the missing phase in the diffraction data. This algorithm has been extensively applied in X-ray and electron diffractive imaging [14-19]. In the HIO algorithm, phase is retrieved to reconstruct the object function via iterations between the real (image) space and the Fourier space with modifications made in each space using 
constraints imposed by the measured diffraction intensities and support for the object [19]. The approach is general without the need for prior information about the structure except the object support.

Iteration in HIO requires forward and backward Fast Fourier Transformation (FFT). However, conventional FFT uses equispaced rectilinear sampling, which cannot be directly extended to tomography reconstruction of diffraction data recorded in a tilt series. To overcome this issue, we use polar FFT as implemented in the general category of non-equispaced FFT (NFFT) methods. The motivation here is to avoid resampling in the diffraction space and its related issues [20]. The basic concept of NFFT computation is to use conventional FFT in a Cartesian grid, while the Fourier frequencies are oversampled and a window function is used to interpolate between equally and non-equally sampled space. The inverse FT is obtained through a least square minimization [21]. In the 3D HIO algorithm using polar FFT, the object function is sampled in a Cartesian grid, while the Fourier space is sampled in the cylindrical grid, where in the xy plane the sampling is in the polar grid with points equally spaced on concentric circles (Fig. 1(a)).
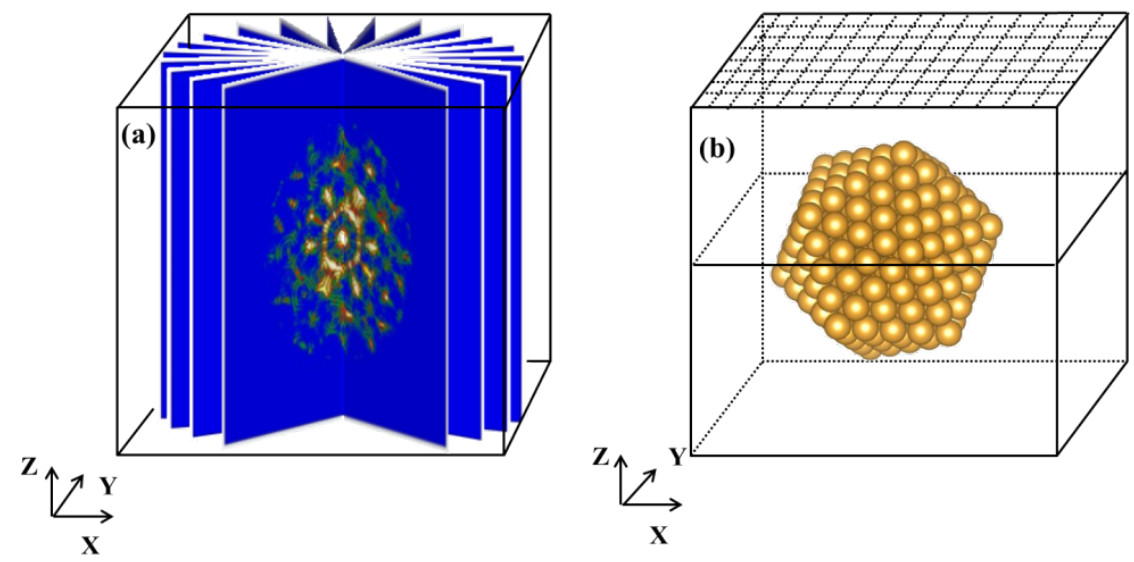

Fig. 1. (a) Sampling in the 3D Fourier space by single-axis tilting Series around the first tilt-axis; (b) 3D object is sampled in the Cartesian coordinate.

For 3D reconstruction, computational cost increases dramatically in order to achieve high resolution. Furthermore, because of oversampling, polar FFT requires a significant increase in the data size, which makes it challenging to implement the HIO algorithm not only in the Fourier space but also in the object space, while maintaining the computational efficiency. We have sought to overcome this challenge using GPU-based acceleration [22, 23], taking advantage of its massively parallel processing architecture.

In what follows, we first describe the methodology of a 3D HIO algorithm based on polar FFT. This is followed by a description of its implementation on GPU. The last section describes a numerical test of our algorithm by reconstructing the 3D structure of an Au icosahedron with 309 atoms using the calculated projected potentials. We analyze the computation performance of our GPU-based algorithm by simulating the noise and missing data wedge in possible experimental situations. Based on the simulation results, we demonstrate the requirements for data sampling, the resolution and the limitation of the method.

\section{GPU Accelerated Hybrid Input-Output Algorithm using Polar FFT}

\subsection{Cylindrical Fourier Transform (CFT)}

Reconstruction of the 3D object using the HIO algorithm is performed in both the Fourier and object spaces. A set of $2 \mathrm{D}$ diffraction patterns are recorded in a tilt series. The diffraction patterns are combined in 3D by taking the rotation axis as the z-axis in the cylindrical coordinate as illustrated in Fig. 1 (a). For each xy plane of constant $\mathrm{z}$, the data is sampled in the polar coordinate of $\vec{k}=(\mathrm{k}, \theta)$ with $k_{n}=n \Delta k$ and $\theta_{m}=m \Delta \theta$. Along $\vec{z}$, the sampling is performed in equal space with $z_{l}=l \Delta z$. Together, the sampling points of $(n, m, l)$ constitute a cylindrical grid. In the object space, a $3 \mathrm{D}$ object is sampled in the equispaced Cartesian coordinate as shown in Fig. 1 (b).

The 3D forward FT is achieved by performing first 2D polar FFT in the xy plane and followed by 1D FFT along z. We call this approach forward cylindrical FT (CFT) in what follows. The 2D polar FFT is applied in each slice of 3D object data (X and Y plane) as step I in Fig. 2. Step II uses regular 1D FFT in the Z dimension of the 3D object data. The 3D inverse FT is carried out by performing 2D inverse polar FT in the xy plane first and then 1D inverse FFT along z. We name it as inverse CFT (CFT'-1) in what follows. 


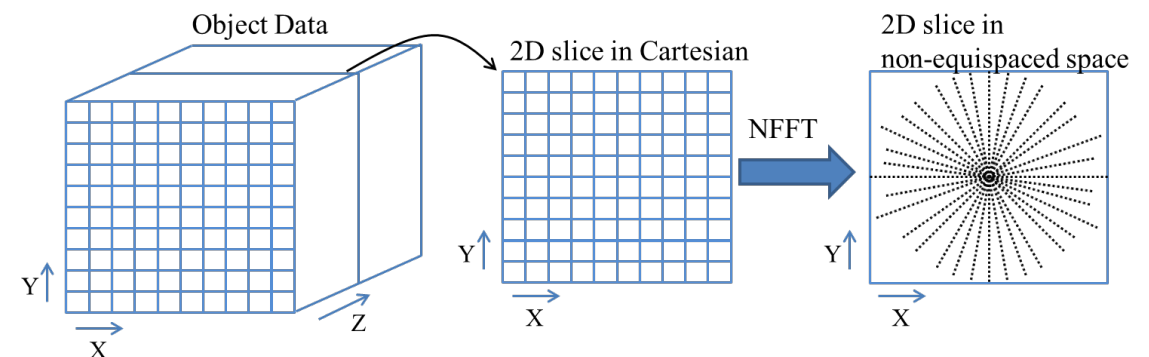

Step I. For each slice, 2D Polar Fourier Transform.

The object is sampled in 3D Cartesian coordinates with equal spaced sampling.

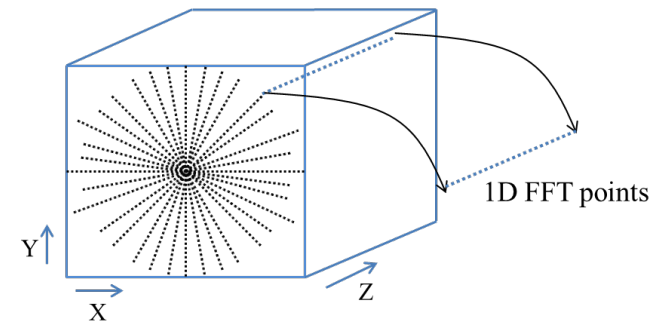

Step II. For each slice, 1D Fourier Transform

Fig. 2. Overview of forward 3D cylindrical Fourier Transform (CFT).

For the 2D polar FFT in the xy plane, let M denote the total number of non-equispaced sampling points in the Fourier space. The points are arranged in a polar grid with number of radial sampling points of $\mathrm{R}$ and number of angular sampling of $\mathrm{T}, R, T \in \mathrm{N}^{*}$ and total sampling points $\mathrm{M}=\mathrm{R} \times \mathrm{T}$. Taking Eq. (1)

$$
\left\{\overrightarrow{\mathrm{k}}_{0}, \overrightarrow{\mathrm{k}}_{1}, \ldots, \overrightarrow{\mathrm{k}}_{\mathrm{M}-2}, \overrightarrow{\mathrm{k}}_{\mathrm{M}-1}\right\}
$$

as the sampling points on the polar grid in the Fourier space, $\mathbb{R}^{2}$ is represented by the unit square $[-1 / 2,1 / 2]^{2}, \overrightarrow{\mathrm{k}}_{\mathrm{j}} \in \mathbb{R}^{2}$ and $\mathrm{j}=0, \ldots, \mathrm{M}-1$. Let $f_{j}$ denote the complex Fourier coefficient at the Fourier spatial frequency $\overrightarrow{\mathrm{k}}_{\mathrm{j}}$. Let Eq. (2)

$$
\mathrm{I}_{N}=\left\{\overrightarrow{\mathrm{r}}_{0}, \overrightarrow{\mathrm{r}}_{1}, \ldots, \overrightarrow{\mathrm{r}}_{\mathrm{N}-2}, \overrightarrow{\mathrm{r}}_{\mathrm{N}-1}\right\}
$$

denotes the set of equispaced Cartesian grid points in the xy plane. For a finite number of complex Fourier coefficients $f_{j}$, the $2 \mathrm{D}$ forward and inverse polar FT can be calculated as

$$
\begin{aligned}
& f_{j}=\sum_{\vec{r}_{n} \in I_{N}} \rho\left(\vec{r}_{n}\right) e^{2 \pi i \vec{k}_{j} \cdot \vec{r}_{n}}, \\
& \rho\left(\vec{r}_{n}\right)=\sum_{\vec{k}_{j} \in \mathbb{R}^{2}} f_{j} e^{-2 \pi \vec{k}_{j} \cdot \vec{r}_{n}} .
\end{aligned}
$$

Details on computation of Eq. (3) as implemented in NFFT can be found in references [24, 25]. The computation involves three basic steps: 1) selection and pre-compute of FT of a window function, 2) fast Fourier transform of $\rho(\vec{r})$ on an over-sampled Cartesian grid, and 3) calculation of the Fourier frequency on the polar grid by interpolation using convolution between the selected window function and the oversampled Fourier frequencies. Step 3 is calculated by using regular FFT. The accuracy of NFFT is adjusted to the requirements by choosing an oversampling factor and a cut-off parameter for the window function, called the convolution kernel width $m$ in NFFT [24]. We employ the NFFT as implemented on GPU in a submitted work [26]. (5):

Inverse 2D polar FT of Eq. (4) is obtained using a least-square approach by solving the unconstrained minimization problem as Eq.

$$
\sum_{j=1}^{M} w_{j}\left|f_{j}-y_{j}\left(\vec{r}_{n}\right)\right|^{2} \stackrel{\tilde{\rho}}{\min },
$$

where

$$
y_{j}=\sum_{\vec{r}_{n} \in I_{N}} \tilde{\rho}\left(\vec{r}_{n}\right) e^{2 \pi \vec{k}_{j} \cdot \vec{r}_{n}}
$$

In Eq. (6), $\tilde{\rho}\left(\vec{r}_{n}\right)$ is the estimated object function. We use the modified conjugated-gradient method as implemented in NFFT for solving the least-square inverse problem. The number of sampling points on the polar grid is forced to be larger than the number of 
points in the Cartersian grid $\left(\mathrm{M} \geq \mathrm{I}_{N}\right)$, so the inverse problem is over-determined. The weight $w_{j}>0$ in the least-square is introduced to account for the sampling density variations in the polar grid. Each step in the iterative optimization process involves a computation of $2 \mathrm{D}$ polar FT. Thus the inverse CFT is computationally intensive [24].

\subsection{ITA using CFT}

The 3D iterative HIO algorithm implemented here follows the same flow as in the previous reported 2D implementation[19] using the methods described previously [13, 15, 16, 18]. Fig. 3 shows a 3D reconstruction algorithm using $2 \mathrm{D}$ tilting serious diffraction pattern images. It is based on iterations between two sets of functions: the real space object function $\rho_{C}$ and its modification $\rho_{C}{ }^{*}$, and the CFT of $\rho_{C}, F_{C}$ and its modification $F_{C} *$. A number $n$ is used to keep track of the iteration.

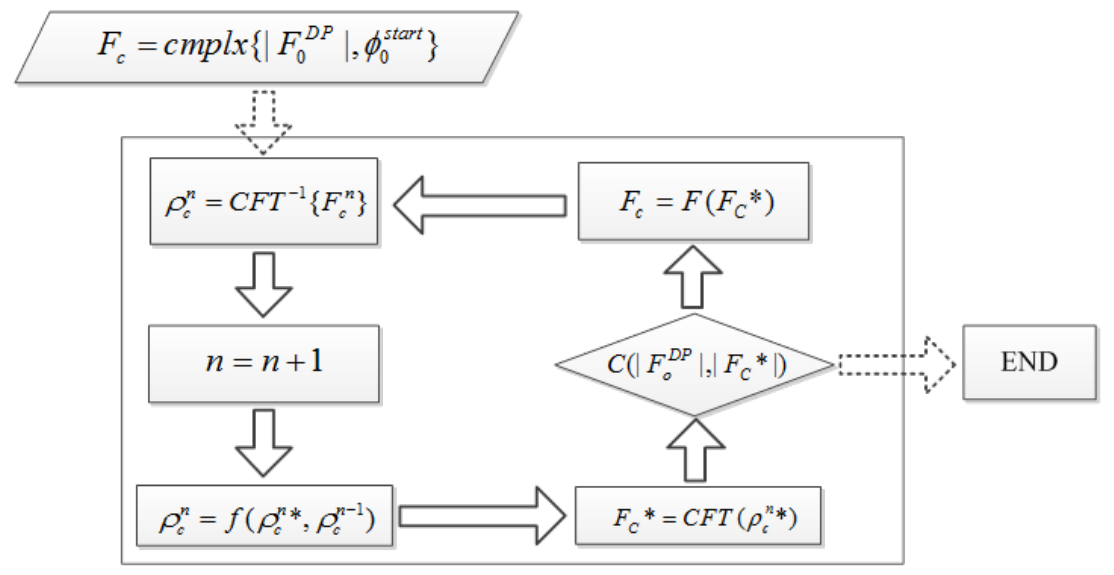

Fig. 3. Iteration algorithm for 3D reconstruction.

The object function $\rho_{C}$ in real space and the $3 \mathrm{D}$ diffraction pattern data set $F_{0}^{D P}$ in reciprocal space are prepared. The starting $\rho_{C}$ is a random function satisfying the object constraints. The object function obtained at the latest iteration is called the current estimate. Critical to reconstruct the object function using the ITA in general are the modifications made during each iteration. The modifications are represented by two functions $\mathrm{f}$ and $\mathrm{F}$ in Fig. 3. The modification function $\mathrm{f}$ is used to change the object function towards a solution that conforms to the known constraints for the object in real space. Similarly, the modification function $\mathrm{F}$ is used to change the Fourier spectrum to conform to the experimental diffraction patterns. There are a number of functional forms of $\mathrm{f}$ and $\mathrm{F}$ that have been proposed; each defines a particular algorithm. Each of these algorithms relies on the available, known real and reciprocal space constraints that can be applied to the object and its Fourier transformation. Here we use the support constraint (S) in $\mathrm{f}$ for an isolated object and modifications as described in the Fienup algorithm for F.

A conditional step is used to terminate the iterative process, when the agreement between the experimental data $F_{0}^{D P}$ and the amplitude of the Fourier transformation of the current estimate meet the criterion. We use the R-factor to measure the percentage of difference between the two arguments agreement here.

\section{GPU Implementation for 3D Reconstruction Algorithm and Its Performance}

We implemented our algorithm on a NVIDIA GPU. To support parallel computing on GPU, NVIDIA designed the Compute Unified Device Architecture (CUDA) as a parallel computing platform and programming model [27]. A general description about GPU computation based on CUDA can be found in the reference [28, 29].

In our 3D HIO algorithm, the computation of 3D inverse CFT, modification in the object space $\left(f\left(\rho_{C}^{n}, \rho_{C}^{n}\right)\right)$, 3D forward CFT and modification in Fourier space $\left(F\left(F_{C}{ }^{*}\right)\right.$ and $\left.C\left(\left|F_{o}^{D P}\right|,\left|F_{C}{ }^{*}\right|\right)\right)$, follow each other in an iteration. Each of these steps involves an operation, or multiple operations, on the 3D data. The 3D data operation is well suited for GPU acceleration. For the modification parts involving $f\left(\rho_{C}^{n}, \rho_{C}^{n}\right), F\left(F_{C}{ }^{*}\right)$ and $C\left(\left|F_{o}^{D P}\right|,\left|F_{C}{ }^{*}\right|\right)$, the parallelization of 3D data point operation is straightforward in CUDA. We use one thread in the GPU to process one data point operation in the 3D data set.

The implementation of 2D NFFT has been reported in a separate paper [26] and an efficient implementation of CUDA 1D FT kernel can be found in CUFFT [30]. An issue arises that the data needed to perform the 1D FT is not stored in CUDA as a contiguous block. To get around this, a temporary array is created to collect these data and store them sequentially.

The algorithm for 3D reconstruction, as presented in Section 2, has been implemented in C programing language. It has been compiled using the gcc compiler version 4.4.5, with the -O3 optimization option, and executed on a workstation equipped with Xeon E5645 (6 physical cores) at 2.40GHz CPU. Our serial CPU implementation of 2D NFFT was adapted from the NFFT3 library[21]. The GPU accelerated codes, implementing the approaches described in Section 2, have been obtained from the serial ones using the CUDA environment for the implementation of parallel tasks and C, and CUDA object files. CUDA codes have been compiled using the NVCC 5.0 compiler and executed on a NVIDIA Tesla C2075 GPU with 14 SMs (each one with 32 CUDA cores at a GPU clock speed of 1.15 $\mathrm{GHz}$ ), equipped with 6GB graphic RAM. Double precision arithmetic has been used for both serial and GPU codes. The parallel GPU version $2 \mathrm{D}$ NFFT is from [26]. In order to quantify the performance enhancement obtained by the GPU based code, we used the performance ratio between the execution times of the CPU and the GPU based codes. 
3D CFT is the part of 3D reconstruction algorithm that requires significant computational power. To evaluate the performance of our GPU reconstruction implementation, we first isolate the 3D CFT GPU implementation into a standalone application and compare execution time with the CPU code implementing. For the 2D NFFT in $\mathrm{x}$ and y plane of CFT, we use the over sampling parameter of $\sigma=2$, which is suggested in literature [31]. In the non-equispaced sampling, cell distribution is in a polar grid and $\mathrm{M}=\mathrm{R} \times \mathrm{T}$ [24]. To evaluate the computing performance in high accuracy, we select convolution kernel of width parameter $\mathrm{m}=7,8,9,10$. The amount of memory on the Tesla M2070 (6 GB) limits the reciprocal space data size $\mathrm{R} \times \mathrm{T} \times \mathrm{Z}$ to no more than $256 \times 180 \times 256$ in double precision on the GPU. Three sets of 3D data were tested: $64 \times 180 \times 64,128 \times 180 \times 128$ and $256 \times 180 \times 256$.

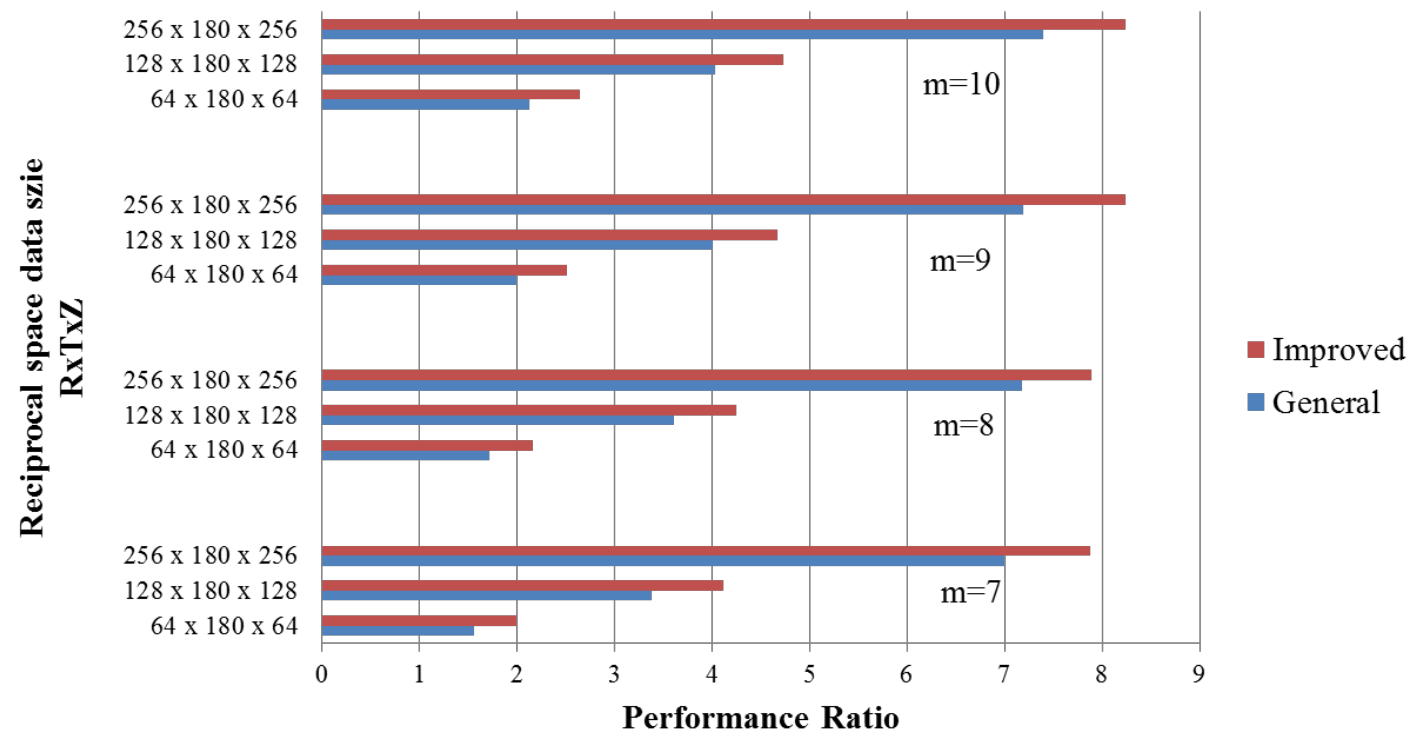

Fig. 4. Performance ratio of GPU CFT and CPU implementation.

Fig. 4 presents the performance ratio of our GPU CFT and CPU implementations in double precision, for different data sizes in nonCartesian space. The polar grid dataset used for the performance evaluation is from the NFFT3 library. For different convolution kernel width, the highest performance ratio obtained is more than 8 times with $\mathrm{m}=9$ and $\mathrm{m}=10$ with the improvements we have made using the temporary array. For different dataset sizes, the performance ratio generally increases as the data size increase. The main reason for the better performance for larger data sets is that the GPU is able to make better use of the computing resources.

Table 1. Execution Time and Performance Ratio between CPU and GPU Implementations

\begin{tabular}{|c|c|c|c|c|c|c|c|c|}
\hline \multirow{2}{*}{$\begin{array}{c}\text { 3D Data size in reciprocal } \\
\text { space }(\mathrm{R} \times \mathrm{T} \times \mathrm{Z})\end{array}$} & \multicolumn{2}{|c|}{ 3D CFT-1 (ms) } & \multicolumn{2}{|c|}{ 3D CFT (ms) } & \multicolumn{2}{|c|}{ Modification partsa (ms) } & \multicolumn{2}{|c|}{ Total (ms) } \\
\hline & $\mathrm{CPU}$ & GPU & $\mathrm{CPU}$ & GPU & $\mathrm{CPU}$ & GPU & $\mathrm{CPU}$ & GPU \\
\hline \multirow{2}{*}{$64 \times 180 \times 64$} & 5007.9 & 1824.5 & 534.6 & 267.8 & 53.85 & 1.82 & 5596.3 & 2094.1 \\
\hline & \multicolumn{2}{|c|}{$2.74 \mathrm{X}$} & \multicolumn{2}{|c|}{$1.99 \mathrm{X}$} & \multicolumn{2}{|c|}{$29.59 \mathrm{X}$} & \multicolumn{2}{|c|}{$2.67 \mathrm{X}$} \\
\hline \multirow{2}{*}{$128 \times 180 \times 128$} & 30025.3 & 5810.8 & 2715.5 & 659.8 & 280.75 & 9.078 & 33047.5 & 6479.6 \\
\hline & \multicolumn{2}{|c|}{$5.17 \mathrm{X}$} & \multicolumn{2}{|c|}{$4.12 \mathrm{X}$} & \multicolumn{2}{|c|}{$30.93 \mathrm{X}$} & \multicolumn{2}{|c|}{$5.1 \mathrm{X}$} \\
\hline \multirow{2}{*}{$256 \times 180 \times 256$} & 216005.5 & 18736.9 & 15528.3 & 1884.3 & 2565.02 & 70.61 & 233498.8 & 20691.9 \\
\hline & \multicolumn{2}{|c|}{$11.52 \mathrm{X}$} & \multicolumn{2}{|c|}{$8.24 \mathrm{X}$} & \multicolumn{2}{|c|}{$36.33 \mathrm{X}$} & \multicolumn{2}{|c|}{$11.28 \mathrm{X}$} \\
\hline \multirow{2}{*}{$512 \times 180 \times 512^{\mathrm{b}}$} & 2165726.7 & 144902.3 & 117465.8 & 5573.5 & 9184.02 & 189.94 & 2292376.5 & 150665.7 \\
\hline & \multicolumn{2}{|c|}{$14.95 \mathrm{X}$} & \multicolumn{2}{|c|}{$21.08 \mathrm{X}$} & \multicolumn{2}{|c|}{$48.35 \mathrm{X}$} & \multicolumn{2}{|c|}{$15.21 \mathrm{X}$} \\
\hline
\end{tabular}

aThe Modification parts include $f\left(\rho_{C}^{n}, \rho_{C}^{n}\right), F\left(F_{C}^{*}\right)$ and $C\left(\left|F_{o}^{D P}\right|,\left|F_{C}{ }^{*}\right|\right)$.

b3D Data size of $512 \times 180 \times 512$ is processed in single precision.

Table. 1 shows the ratio of computing time spent in each of the three main parts (3D $\mathrm{CFT}^{-1}, 3 \mathrm{D}$ CFT, and Modification parts) during reconstruction of different sizes of data sets. The data of the table represents execution time of a single iteration of the reconstruction. Compared to the time spent on $3 \mathrm{D} \mathrm{CFT}-1$ and CFT, the time spent on $f\left(\rho_{C}^{n}, \rho_{C}^{n}\right), F\left(F_{C}^{*}\right)$ and $C\left(\left|F_{o}^{D P}\right|,\left|F_{C}^{*}\right|\right)$ is negligible $($ less than $0.4 \%$ for larger data sizes). The time spent on $\mathrm{CFT}^{-1}$ is much longer than that spent on 3D CFT, making the total runtime almost entirely dependent on the $\mathrm{CFT}^{-1}$ part. Overall, we observe consistent decrease in the computation time using GPU, with the maximum speed-up of more than $36 \mathrm{X}$ in the modification parts and $11 \mathrm{X}$ in $3 \mathrm{D} \mathrm{CFT}^{-1}$ in double precision. For single precision, the total performance ratio can reach $15.21 \mathrm{X}$ with data size $512 \times 180 \times 512$.

\section{Experimental Simulations}


To test the above 3D reconstruction algorithm in a simulated experimental situation, we designed a work flow as shown in Fig. 5. The process consists two parts: data preparation, and reconstruction using iterative transformations. In data preparation, an icosahedral nanocrystal consisting of $309 \mathrm{Au}$ atoms was first built. To acquire tilting series $2 \mathrm{D}$ images, the nanocrystal is rotated along $\mathrm{z}$ axis to angles from 0 to $179^{\circ}$ and projected potential is calculated along each direction. For simplification, we took the simulated image as a convolution between the projected potential and a resolution function. These images are then used as the input files for reconstruction. Thus, our simulations here assume a direct relationship between the projected potential and diffraction intensity. Electron multiple scattering effects are not included here. So the simulation can be said as entirely based on kinematic diffraction.

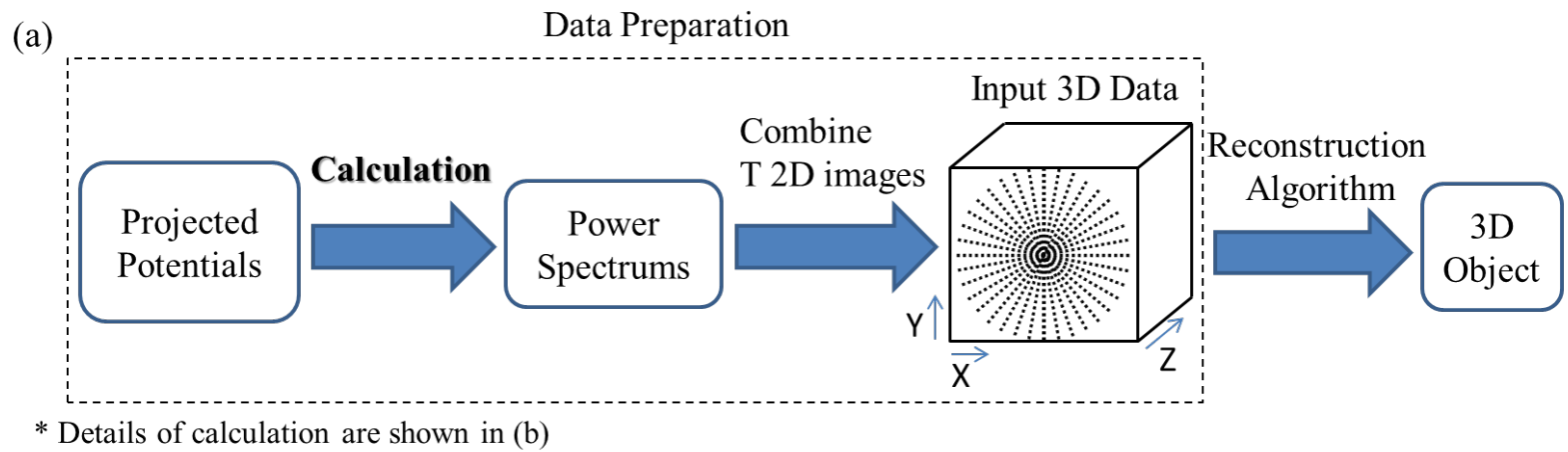

(b)

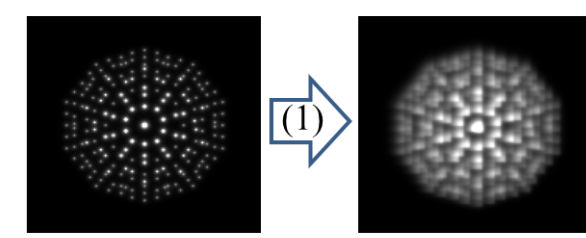

Projected Potential
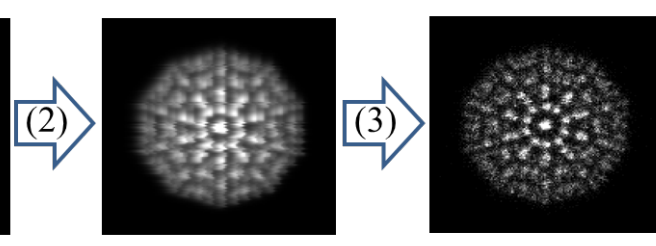

(20)

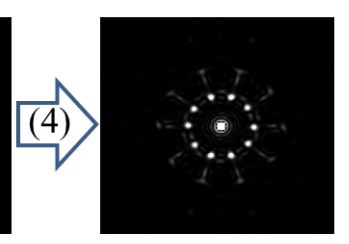

Power Spectra

Fig. 5. The work flow of simulation test. For each wedge, (1) Simulate probe size; (2) Add scanning noise; (3) Add Poisson noise; (4) Calculate Power Spectrum. Here, the probe size is $2 \AA$, scanning noise is 0.5 pixel, Poisson noise $20 \%$.

In the reconstruction part, for each $2 \mathrm{D}$ image, the power spectrum is calculated and stored in the polar grid. The 3D structure of the icosahedral nanoparticle is reconstructed using our algorithm. A typical reconstruction takes about 50 iterations.

To simulate the experimental conditions, we consider the resolution of images as limited by the probe size. Other factors we considered include scanning noise, the signal/noise ratio (SNR) in the recorded image and the range of tilt angles. Fig. 5 (b) demonstrates the 4 steps simulation process. First, the simulated image is convoluted with a resolution function of a specified FWHM. This is followed by adding scanning noise and Poisson noise in the $2^{\text {nd }}$ and $3^{\text {rd }}$ steps. The last step obtains the power spectrum from the simulated image. It should be noted that the simulation process does not include electron multiple scattering, thus, it is only an approximation of the experimental STEM imaging process. In considering the range of tilt angles, we consider the fact that the sample rotation inside TEM is often limited by the size of the pole piece gap, the sample holder design and the sample support. To simulate this, projections within a tilt range, or missing tilting angles in the so called missing wedge, are removed from the reconstruction. In what follows, we present the 3D reconstruction results obtained using simulated data using different levels of resolution, noise, missing wedge and different numbers of $2 \mathrm{D}$ images.

\subsection{Effects of Detector Noise and Scanning Noise}

Here we consider noise in an experiment based on STEM. Noise is introduced during probe scanning and image formation, which includes electron noise and electron detection noise, consisting of detector dark current and readout noise. To simulate this, we start with the calculated projection potentials of the Au icosahedral nanocrystal with $309 \mathrm{Au}$ atoms, which is used as the test object here. An electron probe of $0.1 \mathrm{~nm}$ is simulated using a $2 \mathrm{D}$ Gaussian function with FWHM of $0.1 \mathrm{~nm}$. Images are simulated by convoluting the Gaussian function and the projection potential. Scanning noise is added to the image by randomly by shifting different rows of image with random numbers generated from a Gaussian distribution with the FWHM of $0.024 \mathrm{~nm}$. To simulate the detection noise, the Poisson noise is introduced $[19,32]$. To do this, the image intensity is scaled with intensity at $\lambda$, the noise level is defined by the ratio between its standard deviation and mean, $\sqrt{\lambda} / \lambda$. We used the Poisson noise level from the weakest atom column peak intensity to represent the noise level in the simulated image. To simulate different noise levels, the weakest atom column intensity is scaled to numbers of 50, 25, 10 and 5, for the corresponding noise levels of $14 \%, 20 \%, 33 \%$ and $45 \%$.

After preparing the input images, the same procedure for $3 \mathrm{D}$ reconstruction is followed. With the added noise, the reconstruction took 50 iterations to complete. Fig. 6 shows the reconstructed 3D structures using images with different levels of noise. The noises added to the original projection potential are 14\%,20\%,33\%, and 45\%. The quality of the reconstruction decreases with the level of noise. Nonetheless, single atoms are still distinguishable for all four cases considered here. 


\section{D structure of the icosahedral nanocrystal from selected directions}

Single atom
intensity 5
Noise level: $45 \%$

Single atom
intensity 10
Noise level: $32 \%$

\section{Single atom intensity 25 \\ Noise level: 20\%}

Single atom
intensity 50
Noise level: $14 \%$
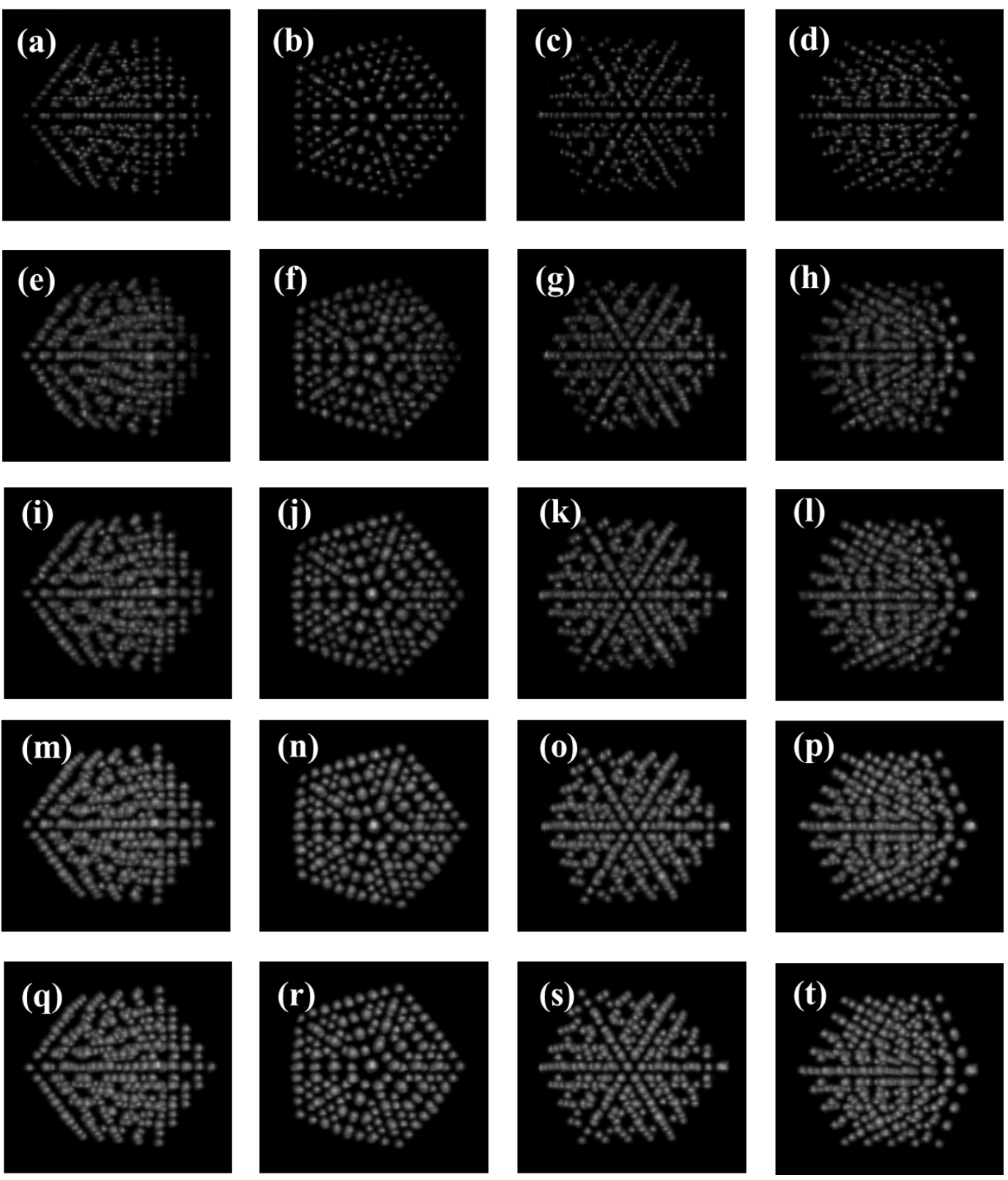

Fig. 6. Comparison of simulations for different noise levels. (a-d) show the reconstructed Au icosahedral particle from four directions the same as those in Fig.6. The input files have a noise level of $45 \%$. In (e-h) the noise level is $32 \%$, and in (i-l) $20 \%$. (m-n) present the reconstruction with a noise level of $14 \%$, which gives the best quality.

\subsection{Effect of Image Resolution}

The point resolution of STEM is determined by the size of the electron probe in STEM experiments. The simulated projection potential has a sampling resolution of $0.02 \mathrm{~nm}$, while experimentally the electron probe obtained in a state of art aberration corrected STEM approaches $0.07 \mathrm{~nm}$. The electron probe could be simulated by a $2 \mathrm{D}$ point spread function with the FWHM determined by the probe size. In our test, the effect of probe size in STEM is simulated by convoluting the projected potential with a Gaussian function. The straightforward result of this convolution is that a point object is now spread in $2 \mathrm{D}$ as a disk, with the intensity peak locating at the original point position. The size of the disk is determined by the FWHM of the Gaussian function, which could be adjusted according to experimental condition.

In this test, the same projection potentials of an $\mathrm{Au}$ icosahedral particle with $309 \mathrm{Au}$ atoms are used. Gaussian functions with different FWHM (full width at half maximum, $2.35 \mathrm{\sigma}$ ) are convoluted with these images. Unlike Poisson noise, whose effect is to add a 
noise background in the diffraction pattern, the convolution of Gaussian function impose a damping envelope in the diffraction pattern, which limits the overall information recorded in diffraction patterns. This becomes the information limit in the reconstruction. 3D reconstruction algorithm could not diminish this effect. The resolution of the $3 \mathrm{D}$ reconstruction is thus limited by the resolution of the $2 \mathrm{D}$ images. 3D reconstructions using $2 \mathrm{D}$ images at different resolution as input are shown in Fig. 7. Atoms remain distinguishable when the probe size approaches $0.2 \mathrm{~nm}$ only if viewing in some certain directions as in Fig. 7 (m-p).

Probe size $0.5 \AA$
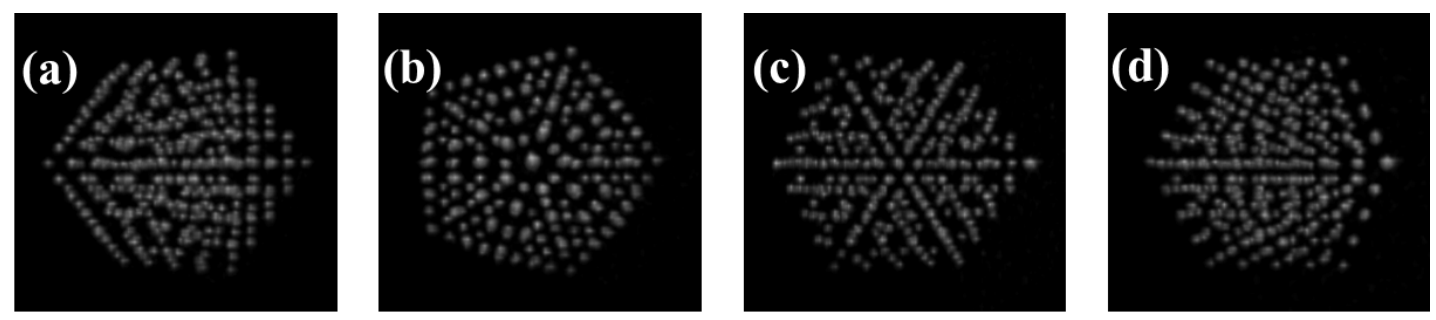

Probe size $1.0 \AA$
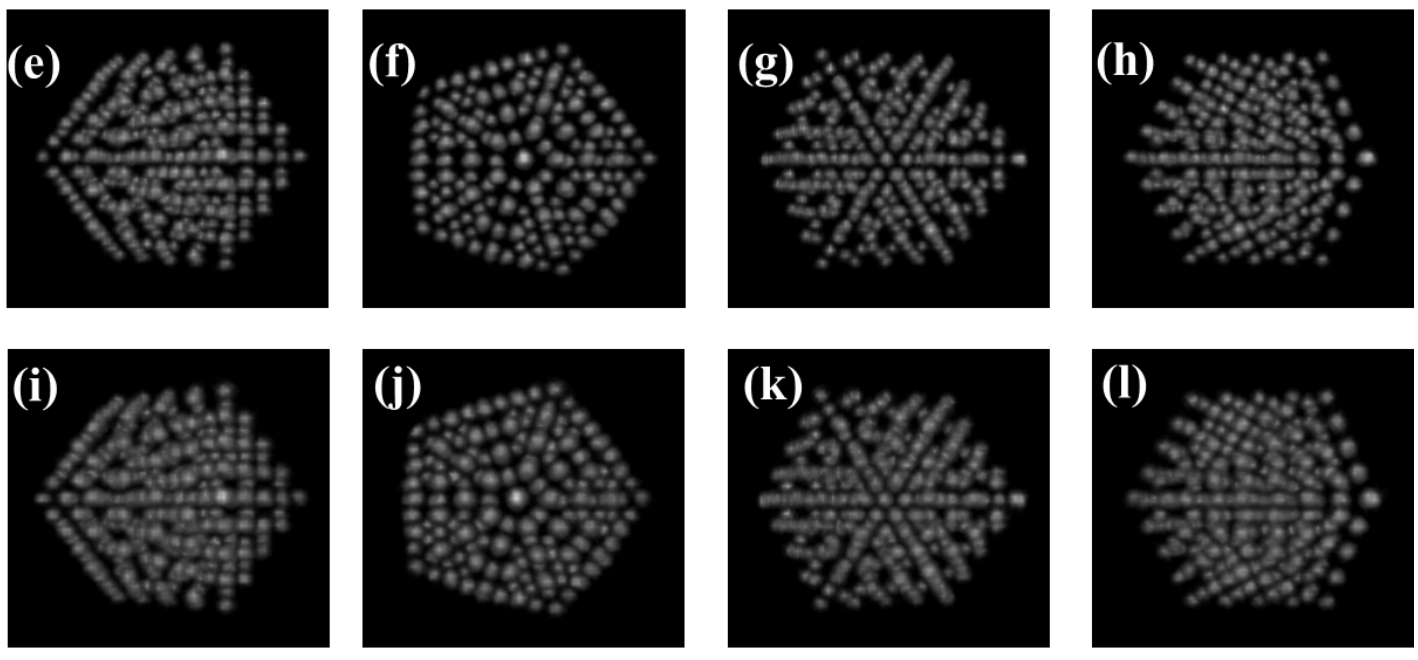

Probe size $1.5 \AA$
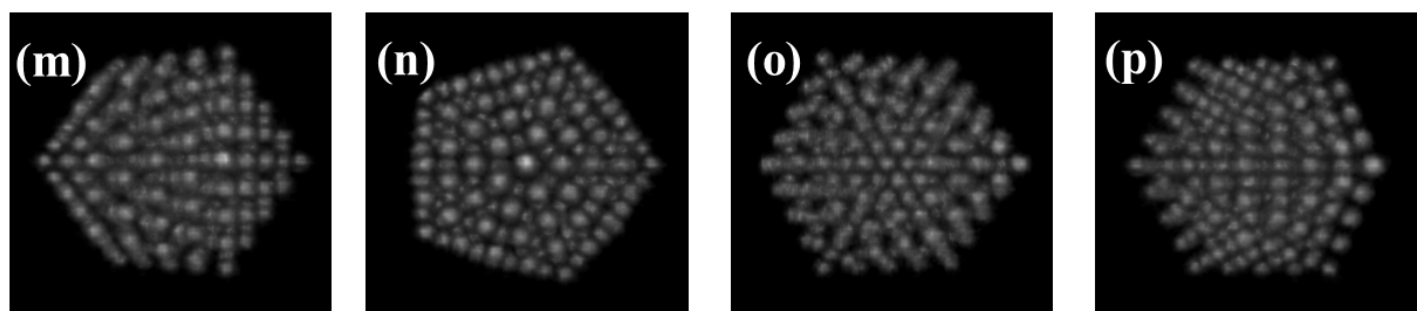

Fig.7. Comparison of simulations for different probe size. From the first row to the last, the probe size used in generating the input files are $0.5 \AA, 1 \AA, 1.5 \AA$ and $2 \AA$ for (a-d), (e-h), (i-l) and (m-n), respectively.

\subsection{Effects of Missing Wedge in Reciprocal Space and Rotation Angle}

Missing a certain range of angles during the tilt-series image acquisition leads to a missing information wedge in the reciprocal space, which is called the 'missing wedge'. In conventional tomography, the missing wedge results in an elongation effect in the reconstructed structure [8]. The iterative transformation algorithms, such as HIO, have capability to retrieve part of missing information in diffraction patterns. For example, previous studies have shown the missing central beam in the diffraction could be reconstructed based on diffraction information recorded in the Bragg diffraction in case of small nanocrystals [33, 34]. Thus, in principle, the $3 \mathrm{D} \mathrm{HIO}$ algorithm here could rebuild the information in the reciprocal space and hence improve the reconstruction result in real space, providing the data acquired in the tilt-series is sufficient for retrieving the missing diffraction information. We tested the tolerance of this algorithm for the missing wedge, the results are described below.

Fig. 8 shows the reconstruction results with different missing wedges. The left row shows the angle range of each missing wedge and its direction in reciprocal space. The atomic structure in 3D remains resolved even when the missing wedge is as large as $30^{\circ}$, shown in $\left(\mathrm{s}^{-} \mathrm{v}\right)$. The information acquired in a tilt series with missing wedge smaller than this limit appears to be sufficient for algorithm to restore the whole 3D structure of the nanoparticle with 309 atoms at atomic resolution. One of the main effects of missing wedge is the shape modification of each atom, shown in the insets in the right column. By specifically examining individual atoms, it is seen that the original sphere turned into a spike as the missing wedge goes larger. The elongation direction of single atoms is along the center direction of each missing wedge. 


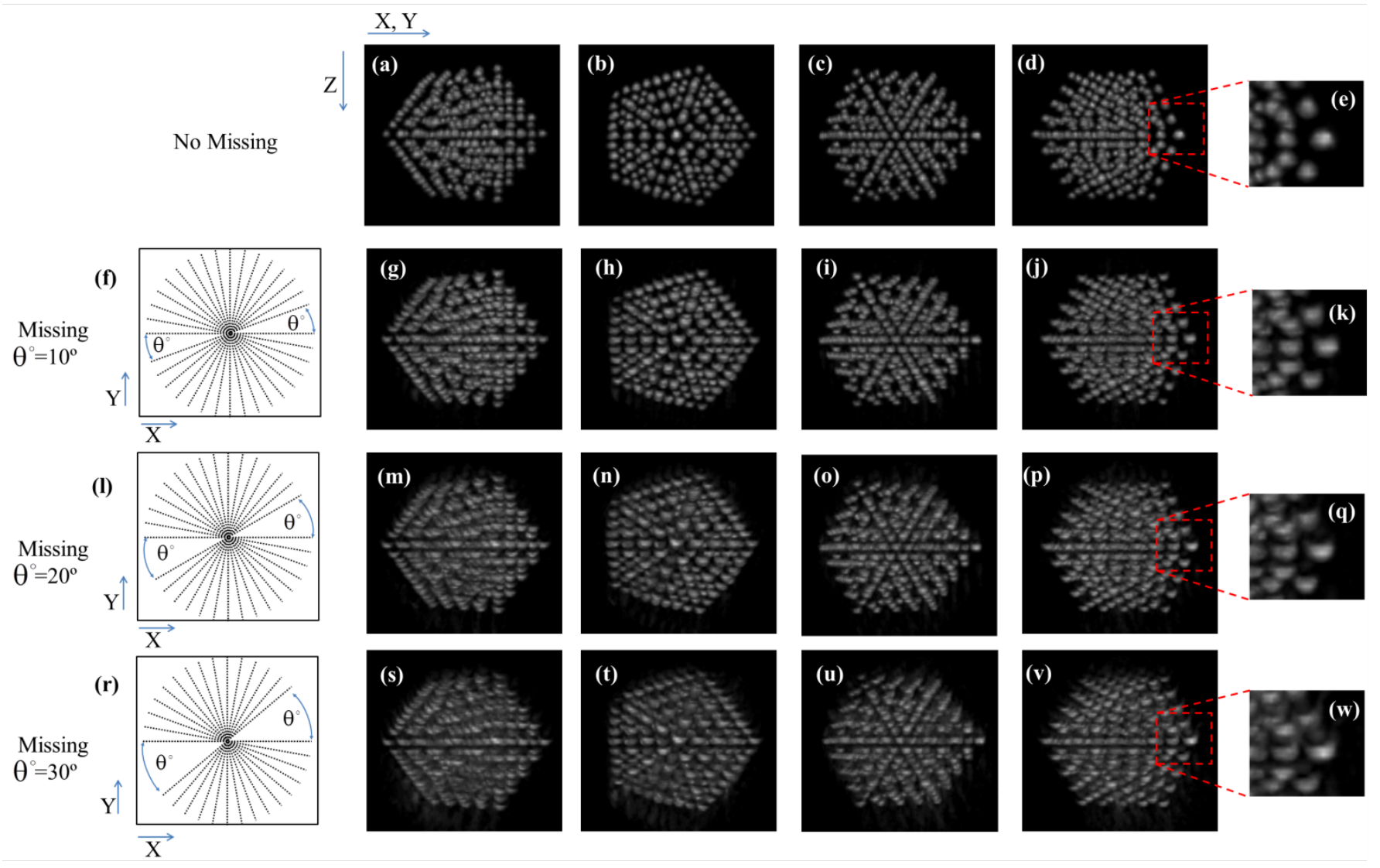

Fig. 8. Comparison of simulations for different values of missing wedge. From the first row to the last, the missing information wedge increases from $0^{\circ}, 10^{\circ}, 20^{\circ}$ to $30^{\circ}$ for $\left(\mathrm{a}^{-\mathrm{d}}\right),\left(\mathrm{g}^{-\mathrm{j}}\right),(\mathrm{m}-\mathrm{p})$ and $\left(\mathrm{s}^{-\mathrm{v}}\right)$, respectively. The missing wedges are shown in the left column of schematics. The (e), (k), (q) and (w) show the zoomed in image of the individual atoms to present the elongation effect.

\subsection{Effects of Rotation Angle Interval}

The above discussion is based on recording tilting series images at $1^{\circ}$ rotation angle interval. To examine the effect of rotation angle interval, we also examined reconstruction using tilt series recorded at $1.5^{\circ}$ and $2^{\circ}$ interval. Experimentally, large rotation interval translates to a smaller number of images required during image acquisition and reduced exposure to the electron beam, which will reduce the amount of sample damage caused by the electron beam. This will potentially benefit the quality of experimental reconstruction. In Fig. 9, three reconstructions using tilting image series with $1^{\circ}, 1.5^{\circ}$ and $2^{\circ}$ intervals are shown in the upper, middle and lower rows, respectively. All input images were prepared using 5\% Poisson noise, 1 A electron probe, scanning noise and $30^{\circ}$ missing wedge to approach experimental condition, which means the first reconstruction (upper row) used 150 images covering the rotation from $0^{\circ}$ to $150^{\circ}$, the second (middle row) used 100 images and the third (lower row) used 75 images covering the same rotation range. Single atoms are resolved with elongation effect in all three cases. With fewer 2D input images, the edge of the atoms becomes blurring due to missing information, which causes intensity overlap. Artifact shadows introduced during the rebuilt appear more obvious outside the particle edges in the lower rows. The blurring of atoms on the edges makes it difficult to observe single atoms in high index orientations. 
150 slices reconstruction
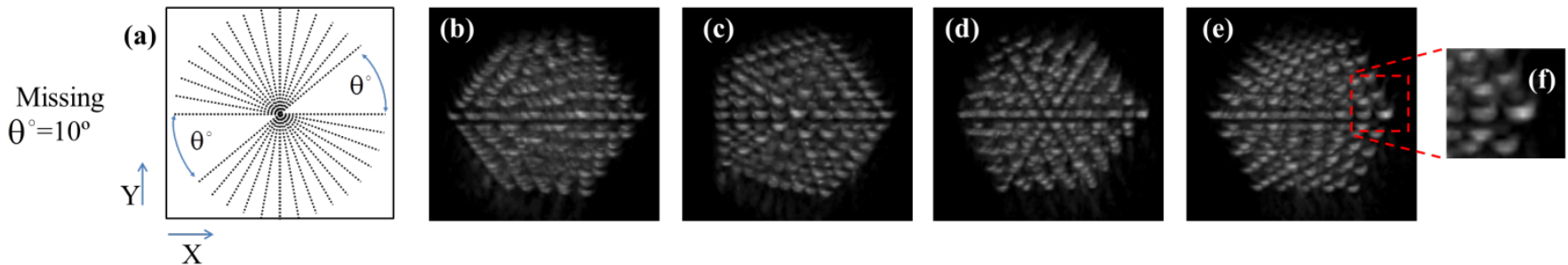

100 slices reconstruction
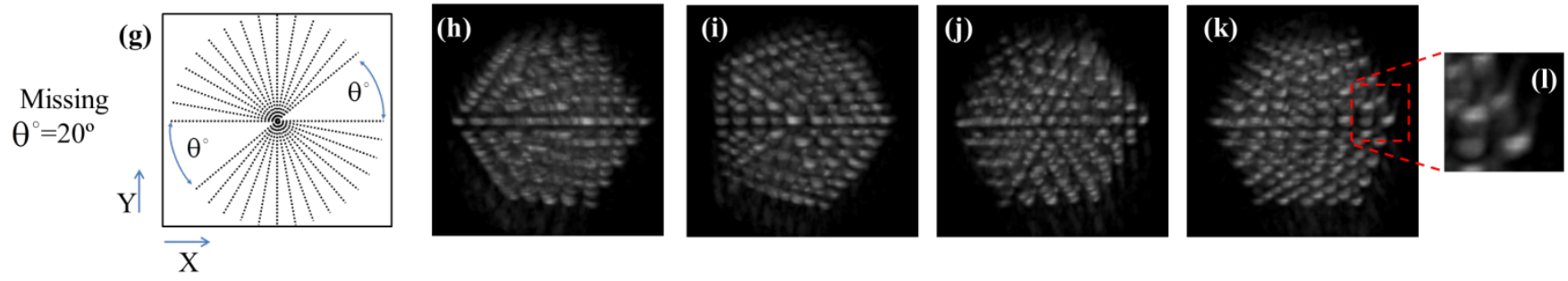

75 slices reconstruction
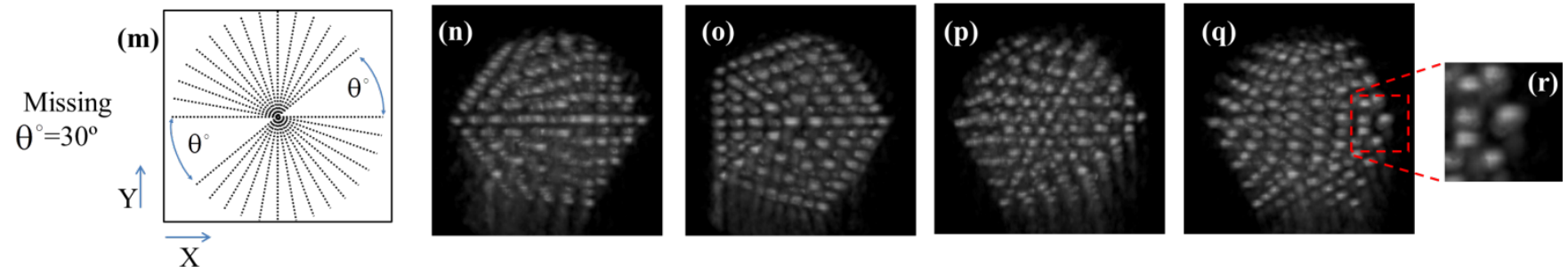

Fig. 9. Comparison of simulations for different rotation interval. From the first row to the last, 150, 100 and 75 slices of $2 \mathrm{D}$ input files are used for the $3 \mathrm{D}$ reconstruction as shown in ( $\mathrm{a}-\mathrm{f}),(\mathrm{g}-\mathrm{l})$ and $\left(\mathrm{m}^{-} \mathrm{q}\right)$, respectively.

\section{Summary and Discussions}

We have described a 3D reconstruction algorithm based on the cylindrical Fourier transform computed by non-equispaced fast Fourier transform. The algorithm is accelerated and implemented using CUDA on GPU. The algorithm was used to test 3D atomic resolution reconstruction for a 309 atoms Au nanoparticle using diffraction patterns calculated from the simulated projected images as input.

Results using simulated test data show that the algorithm is capable of reconstructing the 3D structure at atomic resolution. The reconstructed $3 \mathrm{D}$ object is highly dependent on the quality of the input $2 \mathrm{D}$ diffraction patterns as described by resolution, noise and missing wedge in the simulated image tilt series.

The inverse cylindrical Fourier transform is the most computationally intensive part of the algorithm. By implementing NFFT using the GPU, we achieved a performance ration of 15 compared to the algorithm running on CPU for the dataset of 512x180x512. We expect the benefit of parallel computing using GPU will further increase as the data size increases. Particularly in double precision cases, it can still achieve more than $11 \mathrm{X}$ speed-up.

In Fig. 10, the effects on reconstruction are investigated by plotting the intensity profiles of the projected 2D images. These 2D images are obtained by integrating the intensity from the 3D reconstructed images along the same projection direction. In each image, two intensity profiles along the horizontal and vertical directions are presented. In Fig. 10 (a), the reconstruction uses 180 projected potentials at $1^{\circ}$ rotation angle interval as input files, which is considered as the ideal case. Along the horizontal direction, as shown in Fig. 10 (b), all of the 9 atomic columns are resolved. Among the intensity peak(s) marked by the red arrows, in Fig. 10 (b), the sub peaks convoluted with the main peaks are due to the close distance between two adjacent atomic columns in this projection. Vertically, all four atomic columns are resolved as shown by the four peaks in (c). When reconstruction was performed using the simulated images at $1 \AA$ resolution and with $25 \%$ noise, as shown in Fig. 10 (d-f), the peaks are broadened to $1 \AA$ as measured in term of the FWHM, resulting in the overlap of adjacent atomic peaks, as indicated by the two red arrows in Fig. 10 (e). We also observed an increase in the background intensity from the included $25 \%$ noise, which leads to the lower contrast and the disappearing of weak peaks seen in Fig. 10 (a), as marked by the blue line in Fig. 10 (a), (e) and (h). Vertically, the four columns remains resolved as shown by the major peaks in Fig. 10 (f). With a $30^{\circ}$ missing wedge, we observe a major elongation effect approximately normal to the missing wedge. In Fig. 10 (i), the intensity peak width increase to $2.5 \AA$ as measured by FWHM. This vertical elongation in the image also influences the resolution in horizontal direction. The elongated intensity tails overlap or expand to positions close to other atomic columns, such as the extra peak in (h) indicated by the blue arrow. This peak comes from the intensity tail of the atom column beneath and they are not present in Fig. 10 (b) and (e). Excluding this effect, the resolution remains the same as in Fig. 10 (e) in the horizontal direction, for the case without missing wedge. 

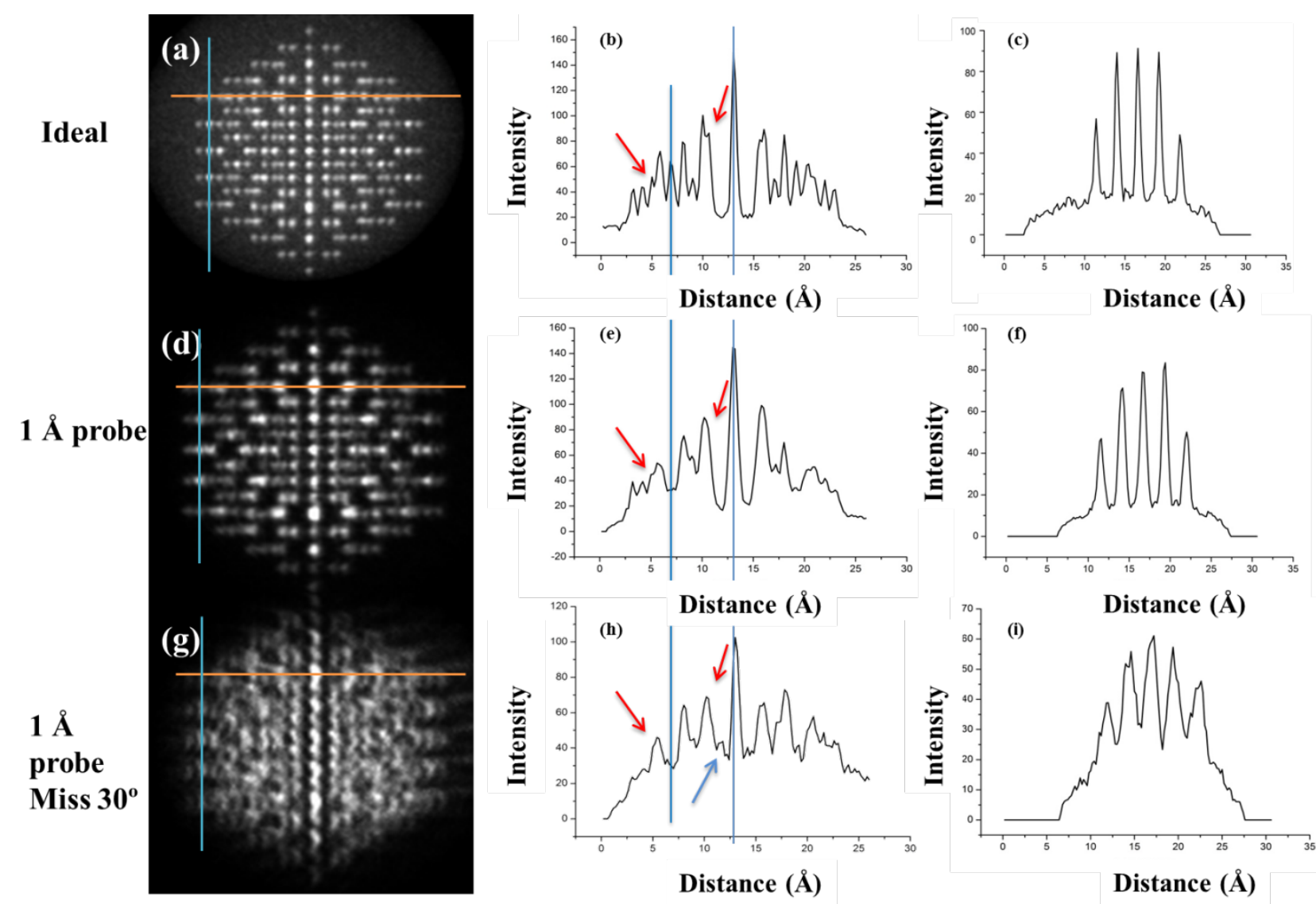

Fig.10. The intensity profiles of the projected $2 \mathrm{D}$ images. Fig (a),(d) and (g) are 2D images obtained by integrating the intensity from the $3 \mathrm{D}$ reconstructed images along the same projection direction for ideal structural model (a), the reconstruction using $1 \AA$ probe (d) and 1 A probe with 30 missing wedge (g). In each image two intensity profiles along the horizontal and vertical directions shown by the orange and blue lines are presented on the right. Figure (b), (e) and (h) are the horizontal profiles and (c), (f) and (i) are the vertical profiles for (a), (d) and (g), respectively.

Thus, without the missing wedge, our algorithm reconstructs the full atomic structure information up to the resolution limit in the simulated images. The missing wedge introduces additional degradation in resolution in the direction normal to the missing wedge. Overall, for the nanoparticle with 309 atoms, our algorithm was capable of reconstructing its 3D structure by resolving individual atoms using simulated atomic resolution projection images, provided that the experimental condition is controlled within the tolerance of $25 \%$ noise level, $1 \AA$ probe formation and sample tilting range of $150^{\circ}$ with $2^{\circ}$ increment. This requirement could be met by or is close to the capability of most STEMs with field emission gun.

It should be emphasized while the degradation in resolution makes the atomic peaks in the $2 \mathrm{D}$ projected hard to distinguish, the reconstructed 3D structures has far more information for the resolving neighboring atoms. The recent demonstration of improved atom detectability using Fourier filtering by Miao[12] is an example. Thus, we anticipate progress in 3D data processing could lead to a lower threshold for reconstructing the $3 \mathrm{D}$ atomic structure.

Our simulation did not take account of the electron multiple scattering effects which likely occur in electron diffraction. Our simulation also did not take account of the limited depth of focus that will be a major issue in STEM when using a large convergence angle. The depth of focus issue can be avoided by recording diffraction patterns directly using a nanometer-sized parallel beam. When applying to real experimental results, in principal, the utilization of diffraction patterns should be able to promote the image quality, because of the higher signal/noise ratio, absence of both objective lens aberration and scanning noise. The issue of electron multiple scattering, in principle, can be taken into account by reconstructing the complex object function. The effectiveness of this approach for small nanoparticles requires further investigations.

\section{Acknowledgement}

This work described here is supported by the US Department of Energy, Division of Materials Science, under Award no. DEFG0201ER45923 and National Natural Science Foundation of China, Award no. 61139002. 


\section{Reference}

[1] P. Batson, N. Dellby, O. Krivanek, Sub-ångstrom resolution using aberration corrected electron optics, Nature, 418 (2002) 617-620.

[2] P.D. Nellist, M.F. Chisholm, N. Dellby, O. Krivanek, M. Murfitt, Z. Szilagyi, A.R. Lupini, A. Borisevich, W. Sides, S.J. Pennycook, Direct sub-angstrom imaging of a crystal lattice, Science, 305 (2004) 1741-1741.

[3] R. Erni, M.D. Rossell, C. Kisielowski, U. Dahmen, Atomic-resolution imaging with a sub-50-pm electron probe, Phys. Rev. Lett., 102 (2009) 096101.

[4] H. Sawada, Y. Tanishiro, N. Ohashi, T. Tomita, F. Hosokawa, T. Kaneyama, Y. Kondo, K. Takayanagi, STEM imaging of 47-pmseparated atomic columns by a spherical aberration-corrected electron microscope with a $300-\mathrm{kV}$ cold field emission gun, J. Electron Microsc., 58 (2009) 357-361.

[5] S. Van Aert, K.J. Batenburg, M.D. Rossell, R. Erni, G. Van Tendeloo, Three-dimensional atomic imaging of crystalline nanoparticles, Nature, 470 (2011) 374-377.

[6] A.Y. Borisevich, A.R. Lupini, S.J. Pennycook, Depth sectioning with the aberration-corrected scanning transmission electron microscope, Proc. Natl. Acad. Sci. U. S. A., 103 (2006) 3044-3048.

[7] A.Y. Borisevich, A.R. Lupini, S. Travaglini, S.J. Pennycook, Depth sectioning of aligned crystals with the aberration-corrected scanning transmission electron microscope, J. Electron Microsc., 55 (2006) 7-12.

[8] H.L. Xin, D.A. Muller, Aberration-corrected ADF-STEM depth sectioning and prospects for reliable 3D imaging in S/TEM, J. Electron Microsc., 58 (2009) 157-165.

[9] R. Ishikawa, A.R. Lupini, S.D. Findlay, T. Taniguchi, S.J. Pennycook, Three-Dimensional Location of a Single Dopant with Atomic Precision by Aberration-Corrected Scanning Transmission Electron Microscopy, Nano Lett., 14 (2014) 1903-1908.

[10] E. Cosgriff, P. Nellist, A Bloch wave analysis of optical sectioning in aberration-corrected STEM, Ultramicroscopy, 107 (2007) 626634.

[11] M.C. Scott, C.-C. Chen, M. Mecklenburg, C. Zhu, R. Xu, P. Ercius, U. Dahmen, B.C. Regan, J. Miao, Electron tomography at 2.4ångström resolution, Nature, 483 (2012) 444-447.

[12] C.C. Chen, C. Zhu, E.R. White, C.Y. Chiu, M.C. Scott, B.C. Regan, L.D. Marks, Y. Huang, J.W. Miao, Three-dimensional imaging of dislocations in a nanoparticle at atomic resolution, Nature, 496 (2013) 74-77.

[13] J.R. Fienup, Phase retrieval algorithms: a comparison, Appl. Opt., 21 (1982) 2758-2769.

[14] J.W. Miao, P. Charalambous, J. Kirz, D. Sayre, Extending the methodology of X-ray crystallography to allow imaging of micrometre-sized non-crystalline specimens, Nature, 400 (1999) 342-344.

[15] U. Weierstall, Q. Chen, J.C.H. Spence, M.R. Howells, M. Isaacson, R.R. Panepucci, Image reconstruction from electron and X-ray diffraction patterns using iterative algorithms: experiment and simulation, Ultramicroscopy, 90 (2002) 171-195.

[16] R.P. Millane, W.J. Stroud, Reconstructing symmetric images from their undersampled Fourier intensities, Journal of the Optical Society of America a-Optics Image Science and Vision, 14 (1997) 568-579.

[17] J.M. Zuo, I. Vartanyants, M. Gao, R. Zhang, L.A. Nagahara, Atomic resolution imaging of a carbon nanotube from diffraction intensities, Science, 300 (2003) 1419-1421.

[18] S. Marchesini, A unified evaluation of iterative projection algorithms for phase retrieval, Review of Scientific Instruments, 78 (2007) 011301.

[19] J.-M. Zuo, J. Zhang, W. Huang, K. Ran, B. Jiang, Combining real and reciprocal space information for aberration free coherent electron diffractive imaging, Ultramicroscopy, 111 (2011) 817-823.

[20] J. Miao, T. Ohsuna, O. Terasaki, K.O. Hodgson, M.A. O'Keefe, Atomic resolution three-dimensional electron diffraction microscopy, Phys. Rev. Lett., 89 (2002).

[21] J. Keiner, S. Kunis, D. Potts, Using NFFT 3---a software library for various nonequispaced fast Fourier transforms, ACM T. Math. Software (TOMS), 36 (2009) 19.

[22] F. Xu, K. Mueller, Accelerating popular tomographic reconstruction algorithms on commodity PC graphics hardware, IEEE T. Nucl. Sci., 52 (2005) 654-663.

[23] F. Xu, K. Mueller, Real-time 3D computed tomographic reconstruction using commodity graphics hardware, Phys. Med. Biol., 52 (2007) 3405.

[24] M. Fenn, S. Kunis, D. Potts, On the computation of the polar FFT, Appl Comput Harmon A, 22 (2007) 257-263.

[25] S. Kunis, S. Kunis, The nonequispaced FFT on graphics processing units, PAMM, 12 (2012) 7-10.

[26] J.B.Y. X. Lu, J. M. Zuo Improved non-equispaced FFT computation with CUDA and GPU, in: Int. J. High Perform. Comput. Appl., 2014.

[27] W.-M. Hwu, C. Rodrigues, S. Ryoo, J. Stratton, Compute unified device architecture application suitability, Comput. Sci. Eng., 11 (2009) 16-26.

[28] D. Kirk, NVIDIA CUDA software and GPU parallel computing architecture, in: ISMM, 2007, pp. 103-104.

[29] D.B. Kirk, W.H. Wen-mei, Programming massively parallel processors: a hands`on approach, Newnes, 2012.

[30] CUFFT-Library 4.1, in, Nvidia, 2014. 
[31] J.I. Jackson, C.H. Meyer, D.G. Nishimura, A. Macovski, Selection of a convolution function for Fourier inversion using gridding [computerised tomography application], IEEE T. Med. Imaging, 10 (1991) 473-478.

[32] G. Haberfehlner, R. Serra, D. Cooper, S. Barraud, P. Bleuet, 3D spatial resolution improvement by dual-axis electron tomography: Application to tri-gate transistors, Ultramicroscopy, 136 (2014) 144-153.

[33] W.J. Huang, R. Sun, J. Tao, L.D. Menard, R.G. Nuzzo, J.M. Zuo, Coordination-dependent surface atomic contraction in nanocrystals revealed by coherent diffraction, Nat. Mater., 7 (2008) 308-313.

[34] W.J. Huang, J.M. Zuo, B. Jiang, K.W. Kwon, M. Shim, Sub-angstrom-resolution diffractive imaging of single nanocrystals, Nat. Phys., 5 (2009) 129-133. 